\title{
Supporting Information: Extension and Limits of Cryoscopy for Nanoconfined Solutions
}

\author{
Benjamin Malfait, ${ }^{\dagger}$ Alban Pouessel,$^{\dagger}$ Aîcha Jani,${ }^{\dagger}$ Denis Morineau ${ }^{\dagger *}$
}

$\dagger$ Institute of Physics of Rennes, CNRS-University of Rennes 1, UMR 6251, F-35042 Rennes, France

\section{Corresponding Author}

* Email: denis.morineau@univ-rennes1.fr 


\section{Phase Diagram and thermograms of Bulk}

\section{Glycerol-Water Solutions}
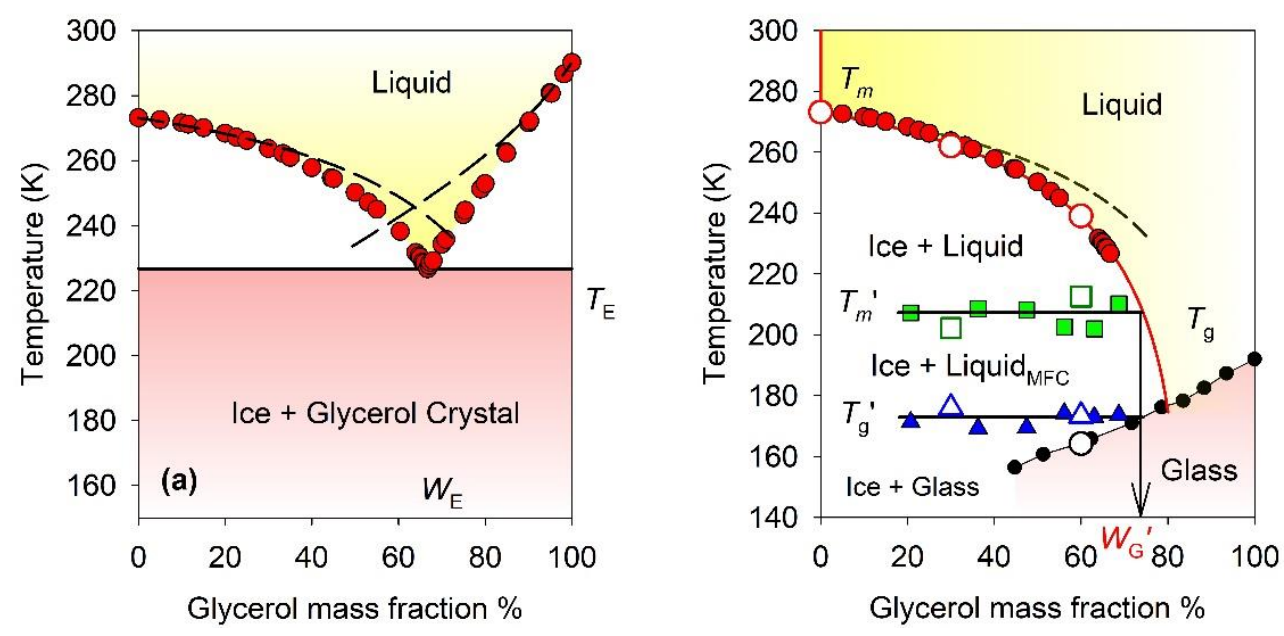

Figure S1. Phase diagram of the glycerol - water binary mixture as a function of the mass fraction of glycerol. (a) Glycerol crystallization was induced by seeding. The experimental equilibrium liquidus lines (filled circles) and eutectic point $\left(T_{\mathrm{E}}\right)$ are from ref. 1. (b) Glycerol did not crystallize (metastable region). The glass transition temperatures of the homogenous aqueous solution $T_{\mathrm{g}}$ (black filled circles) from ref. 2, those of the maximally freeze concentrated solution $T_{\mathrm{g}}$ ' (filled triangles) and the onset of melting of ice in the maximally freeze-concentrated solution $T_{\mathrm{m}}$ ' (filled squares) are from ref. 3. The values obtained from this study (open symbols). The theoretical predictions from ideal mixing are illustrated as dashed lines. Solid lines are guides for the eyes. The composition of the maximally freezeconcentrated solution $\left(W_{\mathrm{G}}{ }^{\prime}\right)$ is indicated by the intercept between $T_{\mathrm{g}}{ }^{\prime}$ and $T_{\mathrm{g}}$. 

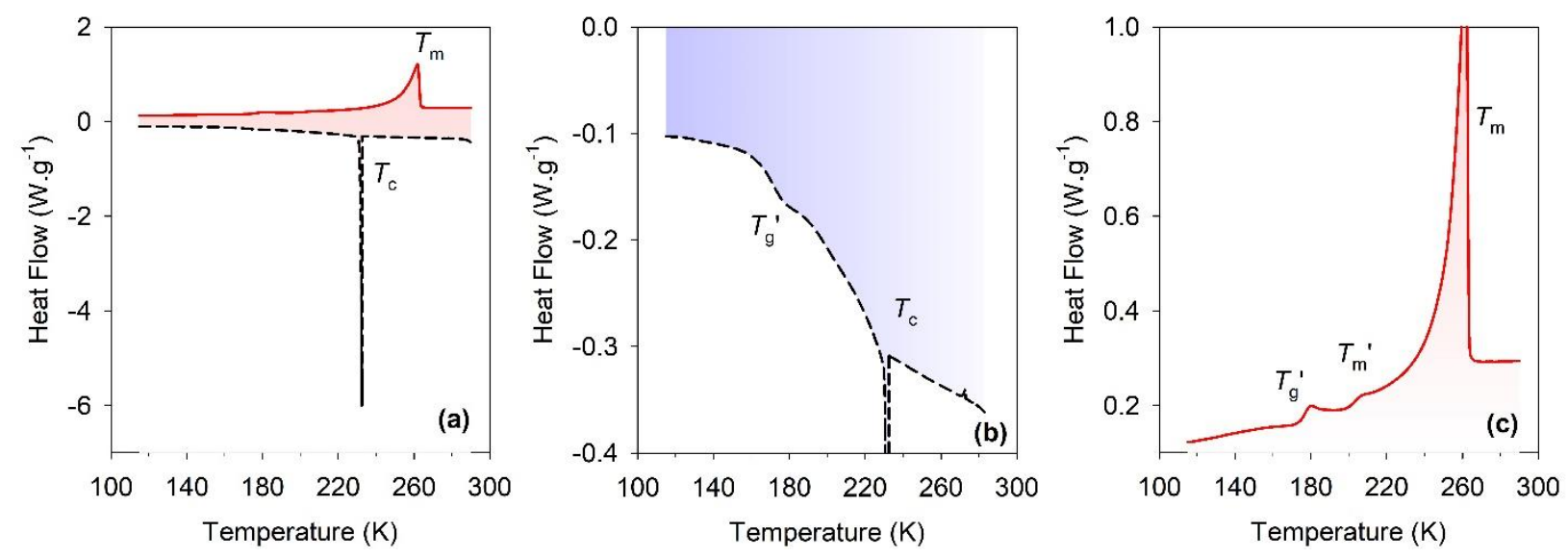

Figure S2. Thermogram of the bulk glycerol solution $\left(W_{\mathrm{G}}=30 \%\right)$. (a) A first cooling ramp from $290 \mathrm{~K}$ to $110 \mathrm{~K}$ (black dashed line) and heating (red solid line), (b) and (c) magnified views of the cooling and heating branches, respectively. 


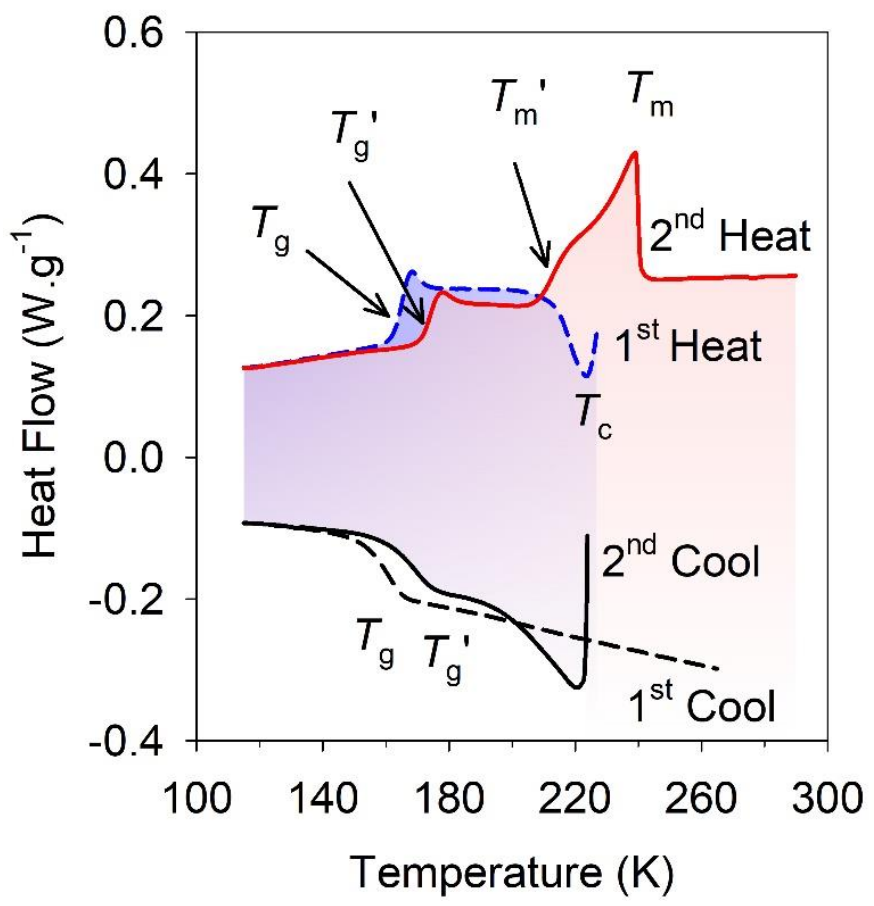

Figure S3. Thermogram of the bulk glycerol solution $\left(W_{\mathrm{G}}=60 \%\right)$ with application of a specific thermal cycling. A first cooling ramp from $290 \mathrm{~K}$ to $110 \mathrm{~K}$ (black dashed line) presents a liquid-to-glass transition at $T_{\mathrm{g}}$, a subsequent first heating ramp up to $227 \mathrm{~K}$ (blue dashed line) presents a glass-to-liquid transition followed by crystallization $\left(T_{\mathrm{c}}\right)$, a second cooling branch down to $110 \mathrm{~K}$ (black solid line) presents a liquid-to-glass transition of the maximally-freezeconcentrated solution at $T_{\mathrm{g}}$, and a final heating ramp up to $290 \mathrm{~K}$ (red solid line) presents the glass-to-liquid transition of the maximally-freeze-concentrated solution at $T_{\mathrm{g}}$, followed by a broad melting peak (onset of melting $T_{\mathrm{m}}$ ' and maximum $T_{\mathrm{m}}$ located at the liquidus). 
2. DSC Thermograms, and Melting Enthalpy of confined Glycerol-Water Solutions 


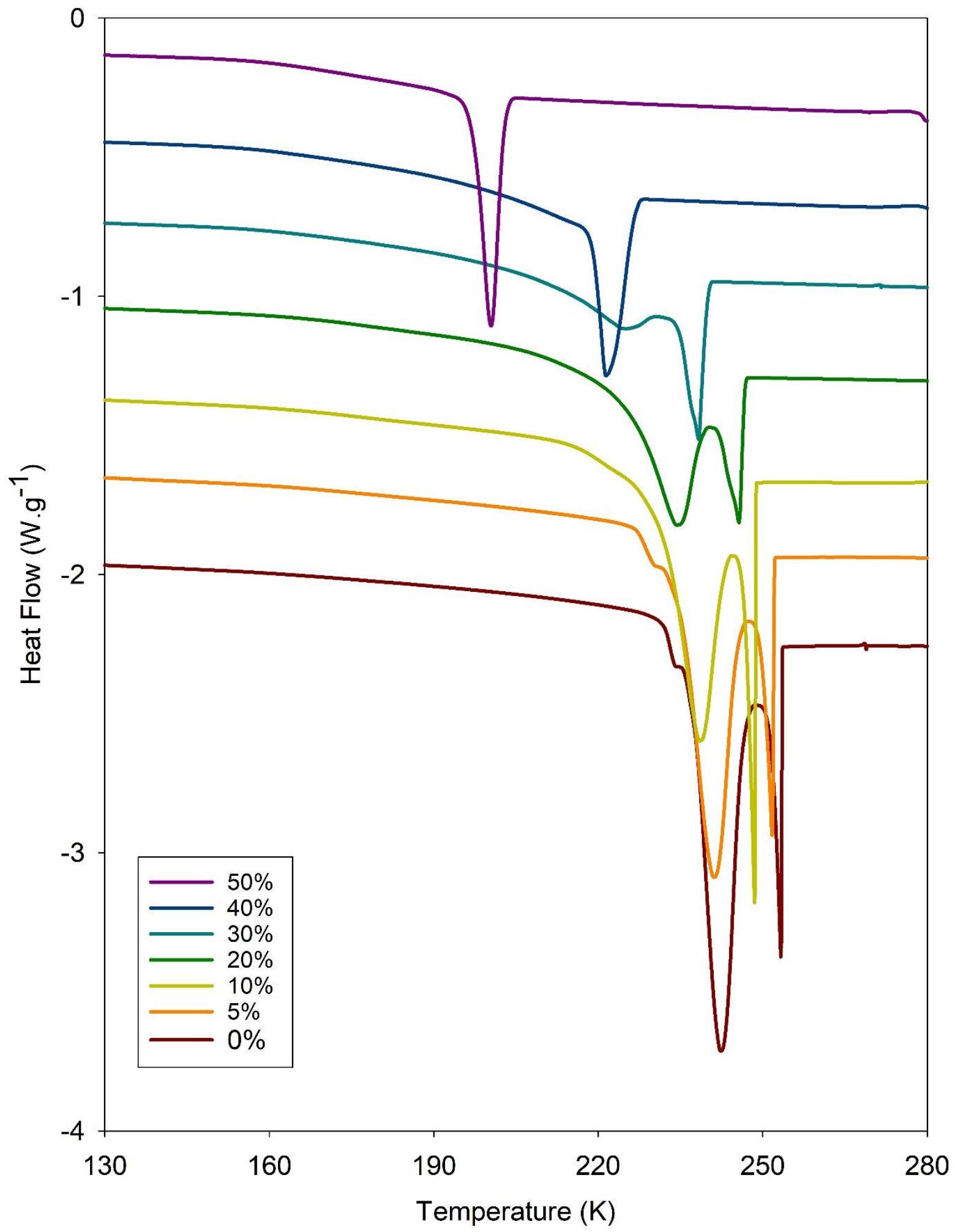

Figure S4 Thermograms of the glycerol solution confined in SBA-15 during cooling from 290 to $115 \mathrm{~K}$. For glycerol mass fractions ranging from 0 to $50 \%$ from bottom to top. Curves are vertically shifted by 0.3 for better clarity. 


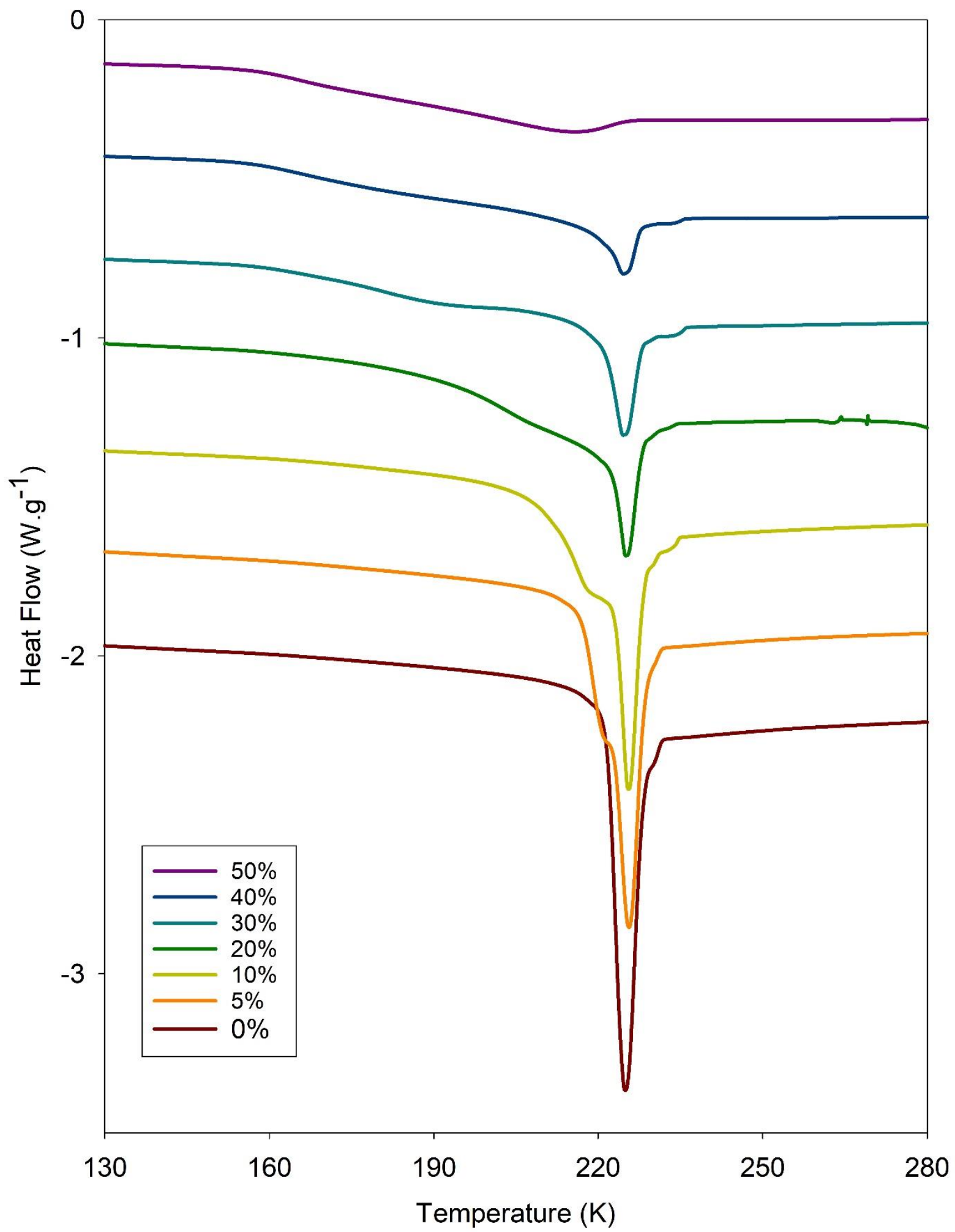

Figure S5 Thermograms of the glycerol solution confined in MCM-41 during cooling from 290 to $155 \mathrm{~K}$. For glycerol mass fractions ranging from 0 to $50 \%$ from bottom to top. Curves are vertically shifted by 0.3 for better clarity. 


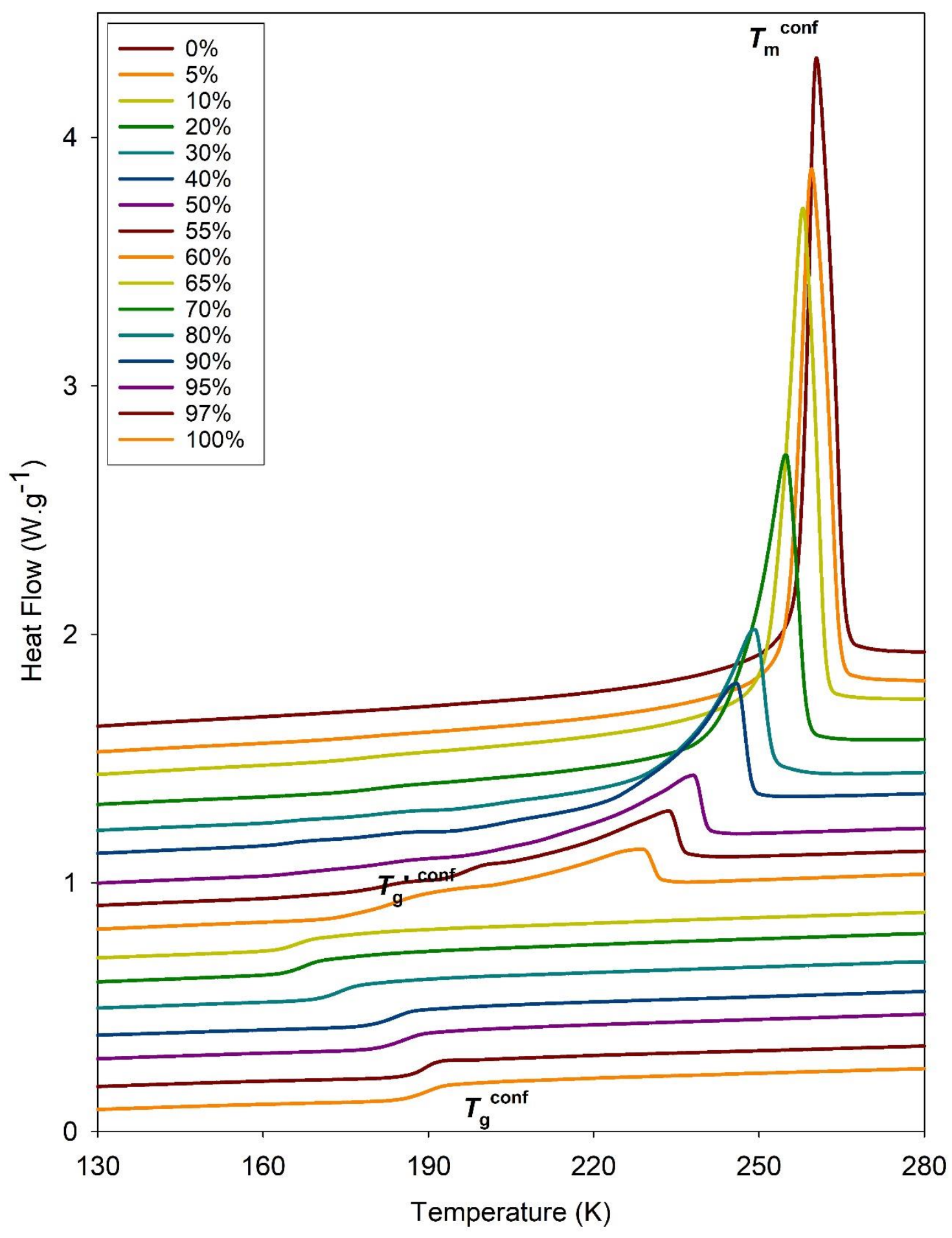

Figure S6. Thermograms of the glycerol solution confined in SBA-15 during heating from 115 to $290 \mathrm{~K}$. Glycerol mass fractions ranging from 0 to $100 \%$ from top to bottom. Curves are vertically shifted by 0.1 for better clarity. 


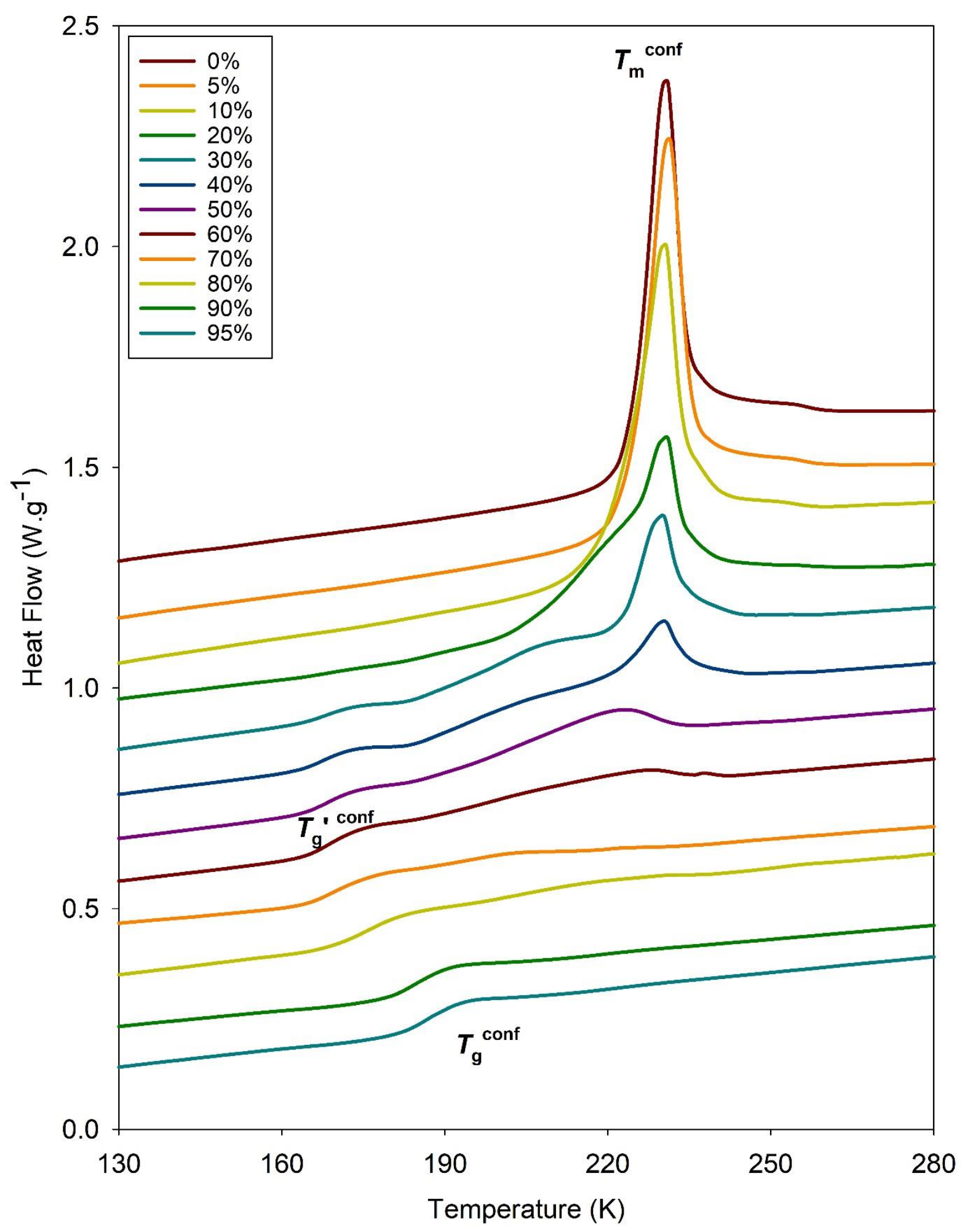

Figure S7. Thermograms of the glycerol solution confined in MCM-41 during heating from 115 to 290K. Glycerol mass fractions ranging from 0 to $95 \%$ from top to bottom. Curves are vertically shifted by 0.1 for better clarity. 


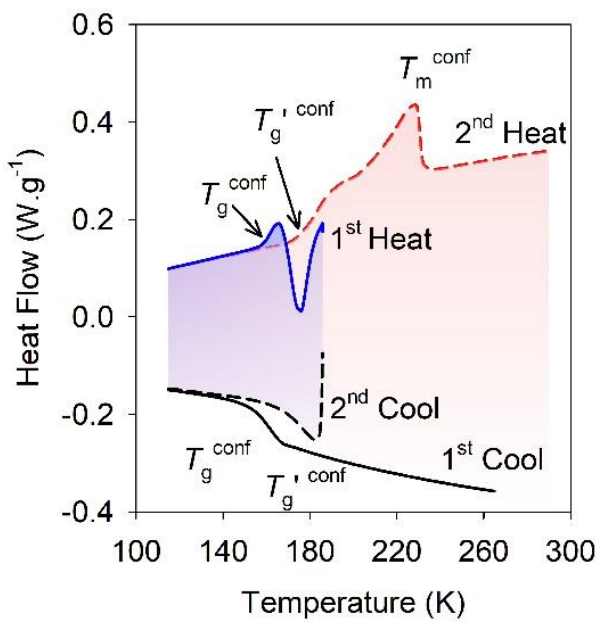

Figure S8. Thermogram of the glycerol solution confined in SBA-15 $\left(W_{\mathrm{G}}=60 \%\right)$ with application of a specific thermal cycling. A first cooling ramp from $290 \mathrm{~K}$ to $115 \mathrm{~K}$ (black solid line) presents a liquidto-glass transition at $\mathrm{T}_{\mathrm{g}}$ conf , a subsequent first heating ramp up to $186 \mathrm{~K}$ (blue solid line) presents a glassto-liquid transition followed by crystallization, a second cooling branch down to $115 \mathrm{~K}$ (black dashed line) presents a liquid-to-glass transition of the maximally-freeze-concentrated solution at $\mathrm{T}_{\mathrm{g}}{ }^{\text {'conf }}$, and a final heating ramp up to $290 \mathrm{~K}$ (red dashed line) presents a broad melting peak.

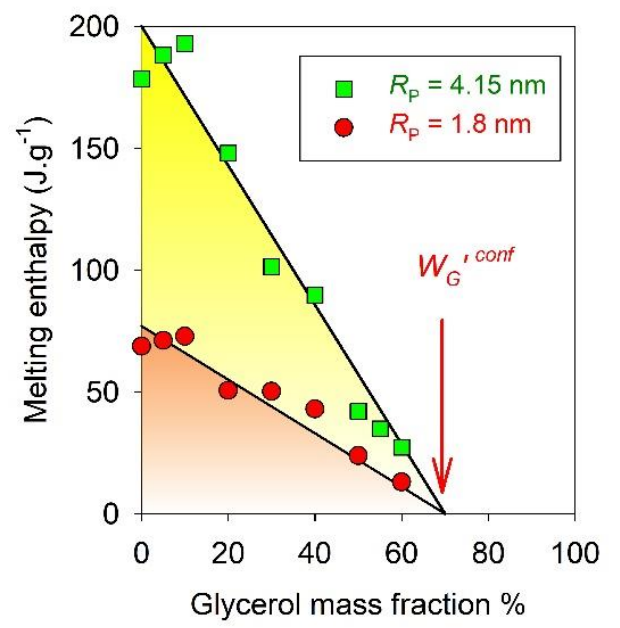

Figure S9. Ice melting enthalpy of the glycerol solutions confined in SBA-15 (green squares) and MCM-41 (red circles) as a function of the glycerol mass fraction $W_{\mathrm{G}}$. The enthalpy was normalized with respect to the mass of the glycerol solution. 


\section{Theoretical Models.}

The Gibbs energy of a crystal of ice, composed of $n_{s}$ water molecules, occupying a cylindrical volume with radius $r$ and length $L$ being contact with a solution of molar concentration $x$ writes as

$$
G_{s}\left(T, n_{s}\right)=n_{s} \mu_{s}(T)+2 \pi r L \gamma_{s l}+2 \pi r^{2} \gamma_{s l}
$$

where $\mu_{s}(T)$ is the chemical potential of water in ice and $\gamma_{s l}$ the ice-solution surface energy.

One notes that

$$
n_{s}=\frac{\pi L r^{2}}{v_{s}}
$$

where $v_{S}$ is the molar volume of water in ice.

From (S1) and (S2) one gets

$$
G_{s}\left(T, n_{s}\right)=n_{s} \mu_{s}(T)+2 \frac{n_{s} v_{s}}{r} \gamma_{s l}+2 \pi r^{2} \gamma_{s l}
$$

The melting temperature $T_{r}^{x}$ of the confined ice of radius $r$ located in a solution of solute molar fraction $x$ is determined by the equilibrium condition between the solid and the liquid phases, which implies that

$$
\left(\frac{\partial G_{s}}{\partial n_{s}}\right)=\left(\frac{\partial G_{l}}{\partial n_{l}}\right)
$$


with $n_{l}$ and $G_{l}$ being respectively the number of water molecules and the total Gibbs energy of the solution that is in equilibrium with the ice crystal. If $r$ is supposed constant, and by definition of $\mu_{l}(T, x)$, the chemical potential of water in the liquid solution, one gets

$$
\begin{gathered}
\mu_{s}\left(T_{r}^{x}\right)+2 \frac{v_{s}}{r} \gamma_{s l}=\mu_{l}\left(T_{r}^{x}, x\right) \\
\mu_{s}\left(T_{r}^{x}\right)-\mu_{l}\left(T_{r}^{x}, x\right)=-\frac{2 v_{s} \gamma_{s l}}{r}
\end{gathered}
$$

The activity of water in a glycerol liquid solution of molar fraction $x$ confined in a pore of radius $r$, at the temperature $T_{r}^{x}$ is defined by

$$
\mu_{l}\left(T_{r}^{x}, x\right)=\mu_{l}\left(T_{r}^{x}, x=0\right)+R T_{r}^{x} \ln \left(a\left(r, T_{r}^{x}, x\right)\right)(\mathrm{S} 7)
$$

where $R$ is the gas constant.

Introducing (S7) in (S6) one gets

$$
\mu_{s}\left(T_{r}^{x}\right)-\mu_{l}\left(T_{r}^{x}, x=0\right)-R T_{r}^{x} \ln \left(a\left(r, T_{r}^{x}, x\right)\right)=-\frac{2 v_{s} \gamma_{s l}}{r}
$$

For the pure confined liquid, on gets

$$
\mu_{s}\left(T_{r}^{0}\right)-\mu_{l}\left(T_{r}^{0}, x=0\right)=-\frac{2 v_{s} \gamma_{s l}}{r}=-\frac{2 v_{s} \gamma_{s l}}{R_{\mathrm{p}^{-}} e}
$$

where $T_{r}^{0}$ is the melting temperature of ice for pure water confined in a pore a radius $R_{\mathrm{p}}$, and $e$ is the thickness of the unfrozen liquid layer, so that $r=R_{\mathrm{p}}-e$.

For the bulk pure liquid, one gets

$$
\mu_{s}\left(T_{b u l k}^{0}\right)-\mu_{l}\left(T_{b u l k}^{0}, x=0\right)=0
$$

where $T_{b u l k}^{0}$ is the melting temperature of bulk pure water. 
For the general case of a confined solution (i.e. eq. S8), it is needed to evaluate, $\mu_{s}\left(T_{r}^{x}\right)-$ $\mu_{l}\left(T_{r}^{x}, x=0\right)$, which is the difference between the chemical potential of pure bulk water in the solid and the liquid states. It could be evaluated by thermodynamical integration between $T_{b u l k}^{0}$ where it vanishes cf. (S10) and $T_{r}^{x}$, noting that for a pure system, the Gibbs-Helmholtz relation applies

i.e. $\left(\frac{\partial\left(\frac{\Delta \mu}{T}\right)}{\partial T}\right)=\frac{-\Delta h}{T^{2}}$, with $\Delta \mu$ and $\Delta h$ being the difference in the chemical potential and molar enthalpy between ice and pure liquid water.

This integration requires a precise knowledge of the temperature variation of the heat capacity of liquid water and ice on a broad temperature range. It has been measured experimentally by DSC by Johari in a temperature range from 273,15 to $153 \mathrm{~K} .{ }^{5}$ The obtained results were later parametrized by Koop at al. ${ }^{6}$, as follows:

$$
\Delta \mu_{0}(T)=\mu_{s}(T)-\mu_{l}(T, x=0)=210368+131.438 T-3.32373 .10^{6} T^{-1}-
$$

$41729.1 \ln (T)$

Neglecting the temperature influence on the heat capacities, classical thermodynamics integration can also be used to give an approximated estimate of $\Delta \mu_{0}(T)$ :

$$
\Delta \mu_{0}(T)=-\Delta H_{m}\left(1-\frac{T}{T_{b u l k}^{0}}\right)-\Delta C_{p}\left(T-T_{b u l k}^{0}\right)+\Delta C_{p} T \ln \left(\frac{T}{T_{b u l k}^{0}}\right)
$$

with $\Delta H_{m}$ and $\Delta C_{p}$ being respectively the molar melting enthalpy and molar heat capacity difference between liquid and ice at the melting point of pure water $T_{b u l k}^{0}$. 
The later equation can be even more simplified, by considering only the first dominant term

as :

$$
\widehat{\Delta \mu}_{0}(T)=-\Delta H_{m}\left(1-\frac{T}{T_{b u l k}^{0}}\right)
$$

Then introducing (S11) in (S8) gives:

$$
\Delta \mu_{0}\left(T_{r}^{x}\right)-R T_{r}^{x} \ln \left(a\left(r, T_{r}^{x}, x\right)\right)=-\frac{2 v_{s} \gamma_{s l}}{r}
$$

Which for the pure confined water is

$$
\Delta \mu_{0}\left(T_{r}^{0}\right)=-\frac{2 v_{s} \gamma_{s l}}{r}
$$

Then assuming that $\gamma_{s l}$ does not vary with $x$ and $T$, subtracting (S15) and (S14) gives

$$
\Delta \mu_{0}\left(T_{r}^{x}\right)-\Delta \mu_{0}\left(T_{r}^{0}\right)-R T_{r}^{x} \ln \left(a\left(r, T_{r}^{x}, x\right)\right)=0
$$

If the limit case of ideal mixing approximation is assumed, i.e. $a\left(r, T_{r}^{x}, x\right)=(1-x)$

(S16) becomes

$$
\Delta \mu_{0}\left(T_{r}^{x}\right)-\Delta \mu_{0}\left(T_{r}^{0}\right)-R T_{r}^{x} \ln (1-x)=0
$$

which allows predicting the cryoscopic melting point depression in confined environment.

If $\widehat{\Delta \mu}_{0}(\mathrm{~S} 13)$ instead of $\Delta \mu_{0}(\mathrm{~S} 11)$ is used to further approximate the chemical potential of pure bulk water, then (S14) writes as (S18), which leads to the extended Gibbs-Thomson equation (S19) as illustrated as dashed line in Figure 2 :

$$
-\Delta H_{m}\left(1-\frac{T_{r}^{x}}{T_{b u l k}^{0}}\right)-R T_{r}^{x} \ln (1-x)=-\frac{2 v_{s} \gamma_{s l}}{r}
$$




$$
T_{r}^{x}-T_{b u l k}^{0}=\frac{-2 v_{s} \gamma_{s l} T_{b u l k}^{0}}{\Delta H_{m} r}+\frac{R T_{r}^{x} T_{b u l k}^{0} \ln (1-x)}{\Delta H_{m}}
$$

For the pure confined water $(x=0)$, the latter gives the classical Gibbs-Thomson equation:

$$
T_{r}^{0}-T_{b u l k}^{0}=\frac{-2 v_{s} \gamma_{s l} T_{b u l k}^{0}}{\Delta H_{m}^{r}}
$$

and for the bulk solution, it gives the classical cryoscopic equation for ideal mixing

$$
\frac{1}{T_{b u l k}^{x}}-\frac{1}{T_{b u l k}^{0}}=-\frac{R \ln (1-x)}{\Delta H_{m}}
$$

Combining the extended Gibbs-Thomson equation for the aqueous solution (S19), and GibbsThomson equation for the pure water (S20) and one can remove explicit reference to the pore size and surface tension, and one gets

$$
T_{r}^{x}=T_{r}^{0}+\frac{R T_{r}^{x} T_{b u l k}^{0} \ln (1-x)}{\Delta H_{m}}
$$

and so the extended cryoscopic equation for ideal mixing

$$
\frac{1}{T_{r}^{x}}-\frac{1}{T_{r}^{0}}=-\left(\frac{T_{b u l k}^{0}}{T_{r}^{0}}\right) \frac{R \ln (1-x)}{\Delta H_{m}}
$$

The predictions from this extended cryoscopic equation for ideal mixing are illustrated as short dashed lines in Figure 4.

In order to quantify the extent of the deviation from the ideal mixing, one can calculate the actual activity of water in the confined mixtures using the experimental values of the melting temperatures by simple transformation of equation (S16), which gives

$$
a\left(r, T_{r}^{x}, x\right)=\operatorname{Exp}\left\{\frac{\Delta \mu_{0}\left(T_{r}^{x}\right)-\Delta \mu_{0}\left(T_{r}^{0}\right)}{R T_{r}^{x}}\right\}
$$

and for bulk liquid 


$$
a\left(r, T_{b u l k}^{x}, x\right)=\operatorname{Exp}\left\{\frac{\Delta \mu_{0}\left(T_{b u l k}^{x}\right)}{R T_{b u l k}^{x}}\right\}
$$

(S24) and (S25) allowed us to evaluate the water activity from the experimental melting points, as shown in Figure 5. 
Table S1. Melting Point of Confined Pure Water ${ }^{\mathrm{a}}$

\begin{tabular}{|l|l|l|}
\hline Pore radius $R(\mathrm{~nm})$ & Experimental melting point $T_{\mathrm{m}}{ }^{\text {conf }}(\mathrm{K})$ & Theoretical melting point $T_{\mathrm{m}}{ }^{\text {conf }}(\mathrm{K})$ \\
\hline 4.15 & 260.4 & 258.5 \\
\hline 1.8 & 230.2 & 229.9 \\
\hline
\end{tabular}

${ }^{a}$ The value of the Gibbs-Thomson constant $C_{G T}=\frac{2 \gamma_{s l} T_{b u l k}^{0}}{\Delta H_{m} \rho_{s}}=52.4 \mathrm{~K} . \mathrm{nm}$ and the thickness of the unfreezable water layer $e=0.6 \mathrm{~nm}$ was used from Findennegg at al. ${ }^{7}$

\section{Notations :}

$r:$ radius of the confined ice

$R_{\mathrm{p}}$ : pore radius

$e:$ thickness of the interfacial liquid layer

$v_{s}:$ molar volume of ice

$\gamma_{s l}:$ ice-liquid surface energy

$L$ : length of the confined ice

$\mu_{s}(T)$ : chemical potential ice at temperature $T$

$\mu_{l}(T, x):$ chemical potential of the solution of composition $x$ at temperature $T$

$\Delta \mu_{0}(T)$ : difference of chemical potential between solid and liquid pure bulk water at temperature $T$.

$T_{\text {bulk }}^{0}:$ melting temperature of bulk pure water 


\section{Materials and Methods}

4.1. Samples. Glycerol (>99\%) were purchased from Sigma-Aldrich and used directly, without further purification. A series of working aqueous solutions of glycerol $(\sim 5 \mathrm{ml}$ each) were prepared by pipetting and addition of deionized water to glycerol. The appropriate value of the composition (in glycerol weight fraction) was checked by measuring the mass at each stage of the preparation. Aqueous solutions were mixed by mechanical agitation until a clear homogeneous liquid phase was obtained and served as stock solutions for the confined systems.

The mesoporous materials MCM-41 silicates were prepared in our laboratory according to a procedure similar to that described elsewhere ${ }^{8}$ and already used in previous works..$^{9,10,11,12}$ Hexadecyl-ammonium bromide was used as template to get a mesostructured triangular array of aligned channels with pore diameter $D=3.65 \mathrm{~nm}$, and porous volume $V_{P}=0.665 \mathrm{~cm}^{3} \mathrm{~g}^{-1}$. The SBA-15 mesoporous silicates were prepared using a procedure described elsewhere, ${ }^{9,13-16}$ with slight modifications of the thermal treatments to optimize the final structure of the product. ${ }^{17}$ Nonionic triblock copolymer (Pluronic $\left.\mathrm{P}_{123}\right)$ : $(\mathrm{EO})_{20}(\mathrm{PO})_{70}(\mathrm{EO})_{20}$ was used as a template to obtain a mesostructured triangular array of aligned channels with a pore diameter $D=8.3 \mathrm{~nm}$, and porous volume $V_{P}=1.0 \mathrm{~cm}^{3} \mathrm{~g}^{-1}$.

Pore size and porous volume were assessed by nitrogen adsorption, and the overall regular porous structure of both matrices were confirmed by transmission electron microscopy and neutron diffraction.

The calcined matrices were dried at $120^{\circ} \mathrm{C}$ under primary vacuum for 12 hours prior to the experiments. The empty MCM-41 and SBA-15 were packed in DSC Tzero@ aluminum hermetic pans and then filled by liquid imbibition with the appropriate weighted amount of 
glycerol aqueous mixtures (typically $3-4 \mathrm{mg}$ ) injected from a syringe to allow complete loading of the porous volume (filling fraction 90-100\%). They were then hermetically sealed in the aluminum pans and their masses measured with a microbalance.

DSC experiments have concluded to the absence of bulk ice crystallization. They indicate that no bulk excess liquid is present out of the matrix, and that the porosity is therefore completely filled, in agreement with previous studies using the same filling method (cf. control of the filling fraction). ${ }^{9,11,12}$

4.2. DSC Experiments. The differential scanning calorimetry (DSC) measurements were performed with a Q-20 TA Instrument equipped with a liquid nitrogen cooling system. The melting transition of an indium sample was used for calibration of temperature and heat flux. The accuracy of the calibration was checked by measuring the melting of pure ice that gave $\mathrm{Tm}=213.17 \mathrm{~K}, \Delta \mathrm{H}_{\mathrm{m}}=310 \mathrm{~J} \cdot \mathrm{g}^{-1}$. The thermograms were recorded with a linear temperature ramp on cooling and heating in the temperature range from 110 to $300 \mathrm{~K}$ (scanning rate of $5 \mathrm{~K} . \mathrm{min}^{-}$ $1)$.

\subsection{Control of the filling fraction}

It is important to note that the control of the filling fraction with no reservoir of excess liquid was confirmed by the absence of additional endothermic peak for all thermograms that would have indicated the melting of a hypothetical excess of water. Moreover, we could demonstrate with samples in which a small amount of excess solution was intentionally used that the excess liquid was detected by an additional peak, precisely located at the bulk melting temperature (cf. Figures S10a and S10b). In addition, we confirmed that in the presence of excess liquid, the melting peak attributed to the confined solution was indeed shifted down to lower temperature (by about $2 \mathrm{~K}$ for $\mathrm{W}=30 \%$, and $7 \mathrm{~K}$ for $\mathrm{W}=60 \%$ ) as illustrated in Figures $\mathrm{S} 11 \mathrm{a}$ and $\mathrm{S} 11 \mathrm{~b}$. This 
effect clearly indicates that the composition of the confined solution differs from the initial one when a reservoir of excess liquid is used. This is consistent with the observed phenomena and the experimental limitations encountered in the literature and discussed in the introductory part of the Letter.
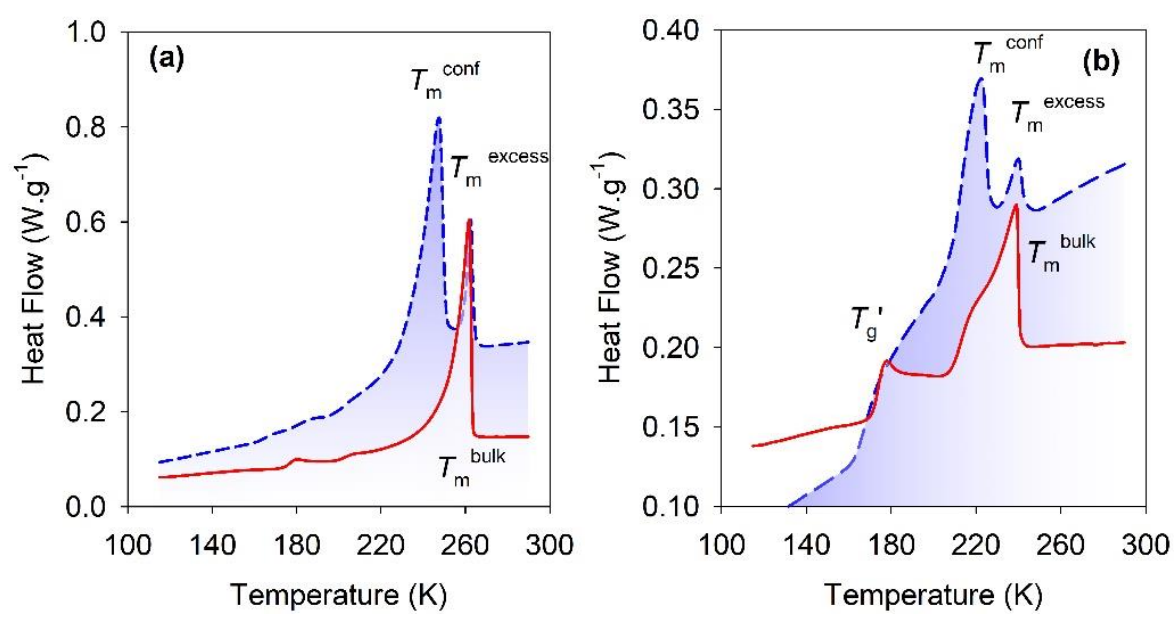

Figure S10. Thermogram during heating of the crystallized bulk solution (red solid line) and confined in SBA-15 (blue dashed line) with an external reservoir of bulk excess liquid. Mass fraction of glycerol (a) $W_{\mathrm{G}}=30 \%$ and (b) $W_{\mathrm{G}}=60 \%$. The thermograms of the bulk solutions are scaled $\mathrm{x} 0.5$ for better clarity.
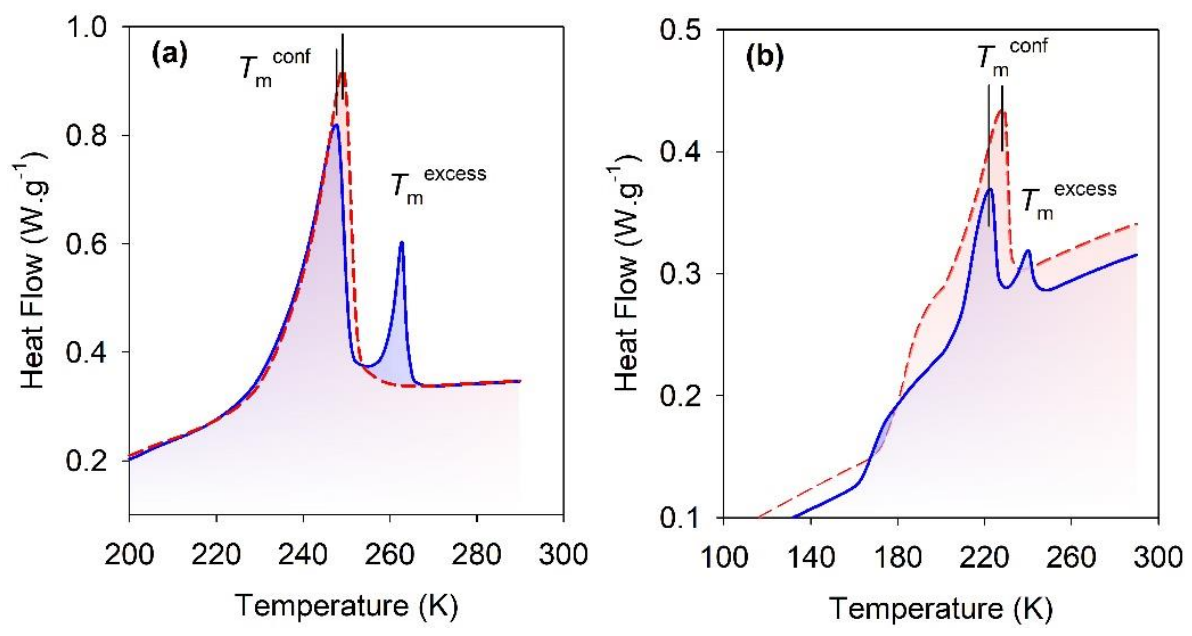
Figure S11. Thermogram during heating of the crystallized solution confined in SBA-15 without (red dashed line) or with (blue solid line) an external reservoir of bulk excess liquid. Mass fraction of glycerol (a) $W_{\mathrm{G}}=30 \%$ and (b) $W_{\mathrm{G}}=60 \%$. The vertical solid lines indicate the downward shift of the melting peak of the confined solution due to the presence of a reservoir of excess liquid.

\section{References}

1. Lane, L., Freezing Points of Glycerol and its Aqueous Solutions. Industrial and Engineering Chemistry 1925, 17, 924-924.

2. Murthy, S.; Singh, G., Examination of the Concentration Dependence of T-g of Binary Aqueous Solutions. Thermochimica Acta 2008, 469 (1-2), 116-119.

3. Bachler, J.; Fuentes-Landete, V.; Jahn, D.; Wong, J.; Giovambattista, N.; Loerting, T., Glass Polymorphism in Glycerol-Water Mixtures: II. Experimental Studies. Physical Chemistry Chemical Physics 2016, 18 (16), 11058-11068.

4. Bachler, J.; Handle, P.; Giovambattista, N.; Loerting, T., Glass Polymorphism and Liquid-Liquid Phase Transition in Aqueous Solutions: Experiments and Computer Simulations. Physical Chemistry Chemical Physics 2019, 21 (42), 23238-23268.

5. Johari, G. P.; Fleissner, G.; Hallbrucker, A.; Mayer, E., Thermodynamic Continuity between Glassy and Normal Water. Journal of Physical Chemistry 1994, 98 (17), 4719-4725. 6. Koop, T.; Luo, B. P.; Tsias, A.; Peter, T., Water Activity as the Determinant for Homogeneous Ice Nucleation in Aqueous Solutions. Nature 2000, 406 (6796), 611-614. 
7. Findenegg, G. H.; Jahnert, S.; Akcakayiran, D.; Schreiber, A., Freezing and Melting of Water Confined in Silica Nanopores. Chemphyschem 2008, 9 (18), 2651-2659.

8. Grun, M.; Lauer, I.; Unger, K. K., The Synthesis of Micrometer- and SubmicrometerSize Spheres of Ordered Mesoporous Oxide MCM-41. Advanced Materials 1997, 9 (3), 254$\&$.

9. Alba-Simionesco, C.; Dosseh, G.; Dumont, E.; Frick, B.; Geil, B.; Morineau, D.; Teboul, V.; Xia, Y., Confinement of Molecular Liquids: Consequences on Thermodynamic, Static and Dynamical Properties of Benzene and Toluene. European Physical Journal E 2003, $12(1), 19-28$.

10. Morineau, D.; Dosseh, G.; Alba-Simionesco, C.; Llewellyn, P., Glass Transition, Freezing and Melting of Liquids Confined in the Mesoporous Silicate MCM-41.

Philosophical Magazine B 1999, 79 (11-12), 1847-1855.

11. Morineau, D.; Xia, Y. D.; Alba-Simionesco, C., Finite-Size and Surface Effects on the Glass Transition of Liquid Toluene Confined in Cylindrical Mesopores. Journal of Chemical Physics 2002, 117 (19), 8966-8972.

12. Morineau, D.; Guegan, R.; Xia, Y.; Alba-Simionesco, C., Structure of Liquid and Glassy Methanol Confined in Cylindrical Pores. Journal of Chemical Physics 2004, 121 (3), 1466-1473.

13. Zhao, D.; Huo, Q.; Feng, J.; Chmelka, B.; Stucky, G., Nonionic Triblock and Star Diblock Copolymer and Oligomeric Surfactant Syntheses of Highly Ordered, Hydrothermally Stable, Mesoporous Silica Structures. Journal of the American Chemical Society 1998, 120 (24), 6024-6036.

14. Audonnet, F.; Brodie-Linder, N.; Morineau, D.; Frick, B.; Alba-Simionesco, C., From the Capillary Condensation to the Glass Transition of a Confined Molecular Liquid: Case of Toluene. Journal of Non-Crystalline Solids 2015, 407, 262-269. 
15. Dosseh, G.; Brodie-Linder, N.; Frick, B.; Le Quellec, C.; Morineau, D.; AlbaSimionesco, C., Dynamical Properties of Toluene and ortho-Terphenyl Confined in MCM-41 and SBA-15 Mesoporous Materials. Annales De Chimie-Science Des Materiaux 2005, 30 (4), 365-373.

16. Xia, Y. D.; Dosseh, G.; Morineau, D.; Alba-Simionesco, C., Phase Diagram and Glass Transition of Confined Benzene. Journal of Physical Chemistry B 2006, 110 (39), 19735-19744.

17. Brodie-Linder, N.; Dosseh, G.; Alba-Simonesco, C.; Audonnet, F.; Imperor-Clerc, M., SBA-15 synthesis: Are there Lasting Effects of Temperature Change within the First 10 min of TEOS Polymerization? Materials Chemistry and Physics 2008, 108 (1), 73-81. 